\title{
Effect of impact angles on ejecta and crater shape of aluminum alloy 6061-T6 targets in hypervelocity impacts
}

\author{
M. Nishida a , K. Hayashi, and Y. Ito \\ Nagoya Institute of Technology, Gokiso-cho, Showa-ku, Nagoya 466-8555, Japan
}

\begin{abstract}
The effect of the impact angle of projectiles on the crater shape and ejecta in thick aluminum alloy targets was investigated in hypervelocity impacts. When polycarbonate projectiles and aluminum alloy 6061-T6 target were used, the impact angle of the projectiles clearly affected the crater shape, as expected. The impact angle also affected the ejecta mass, ejecta size and scatter angle. However, the effect at $15^{\circ}$ and $22.5^{\circ}$ was not great. When the impact angles were $30^{\circ}$ and $45^{\circ}$, the effect was clearly confirmed. The impact angle clearly affected the axial ratio of ejecta fragments, $c / a$.
\end{abstract}

\section{Introduction}

Space debris orbits in a low Earth orbit (LEO) at velocities of more than several km/s, and often strikes spacecraft and space stations. The International Space Station is equipped with shields such as the stuffed Whipple bumper to protect it against space debris.

Small-sized space debris, which has low kinetic energy, does not perforate the shields of space stations or the outer surfaces of spacecraft and space stations but forms craters on these surfaces. In such cases, materials from the target surface are ejected, and the projectile fragments are widely scattered. These fragments and ejected materials become new debris, as pointed out by Murr and his coworkers [1]. They studied the hypervelocity impacts of projectiles on thick targets, and examined the crater formation and impact fragmentation of projectiles experimentally and numerically [2]. The international standardization of test procedures to evaluate spacecraft material ejecta is being promoted [3]. Many studies have been conducted on related phenomena $[4,5]$. However, very few studies have been conducted on crater formation and ejecta composition when projectiles strike thick targets [6], whereas numerous studies have analyzed the impacts of projectiles on thin plates.

In this study, we investigated the effects of the impact angle of very high velocity projectiles on crater size and ejecta in thick aluminum alloy targets. The diameter and depth of the crater after impact were measured. The ejecta collected from the test chamber were examined in detail, and the witness plates were also observed.

\section{Experimental methods}

Projectiles with a diameter of $14.4 \mathrm{~mm}$ made of polycarbonate $(1.82 \mathrm{~g})$ were accelerated using a two-stage light gas gun at Nagoya Institute of Technology. The impact velocity was approximately $1.8 \mathrm{~km} / \mathrm{s}$. We used thick targets (95-mm diameter, 20-mm thickness) made of aluminum alloy 6061-T6 with two cut-off surfaces, as shown in Fig. 1; the creation of cut-off surfaces provided additional

a e-mail: nishida.masahiro@nitech.ac.jp stability. Aluminum alloy 6061 is a precipitation hardened aluminum alloy, containing magnesium and silicon as its major alloying elements, and it has good mechanical properties. Aluminum alloy 2219-T87 is used in solid rocket boosters, space station walls and so on. However, it is difficult to purchase thick plates made of aluminum alloy 2219-T87. Instead, aluminum alloy 6061-T6 was used for the impact study.

The effects of the impact angle $\left(\alpha=0^{\circ}, 15^{\circ}, 22.5^{\circ}\right.$, $30^{\circ}, 45^{\circ}$ and $60^{\circ}$ from normal impact) of projectiles on crater size and ejecta from targets were examined. The mechanical properties of the projectile materials are listed in Table 1. Four witness plates $(240 \mathrm{~mm} \times 210 \mathrm{~mm}$ and $50 \mathrm{~mm} \times 235 \mathrm{~mm}, 2 \mathrm{~mm}$ in thickness) made of copper, $\mathrm{C} 1100 \mathrm{P}-1 / 4 \mathrm{H}$, with a hole were placed $50 \mathrm{~mm}$ in front of each target as shown in Fig. 1. In order to examine the effect of impact angle, the witness plates and targets were rotated together. Therefore, the front witness plate (the largest witness plate) and target were always parallel. As a result, the holes through which the projectiles passed were not located in the center of the witness plates and were located on the trajectory of the projectiles. The size of the hole was $22.0 \mathrm{~mm}$ in length and $22.0 \mathrm{~mm}$ to $47.5 \mathrm{~mm}$ in width, depending on impact angle.

\section{Results and discussion}

\subsection{Crater size}

Figure 2(a)-(f), containing photographs of the craters, shows the effect of impact angle. The projectiles came from the left side of each image and were reflected toward the right side. When $\alpha=0^{\circ}$ (normal impact to target), the crater lips were not long, and appeared broken. The crater edge was rough. Aluminum alloy 6061-T6 has larger yield stress, tensile strength and hardness and smaller elongation at break, compared with pure aluminum alloys, e.g. the 1000 series. Reflecting such mechanical properties, the large crater lips were not formed and the crater edge was rough [7].

As the impact angle increased, the height of the crater lips decreased, and when $\alpha=45^{\circ}$ and $60^{\circ}$ the crater lips became indistinctive. Instead, dark streaks and spray were 
Table 1. Mechanical properties of aluminum alloy 6061-T6 obtained by static tensile tests and hardness tests.

\begin{tabular}{lc}
\hline Tensile Strength [MPa] & 322 \\
\hline Yield Stress [MPa] & 287 \\
\hline Elongation at break [\%] & 9 \\
\hline Vickers Hardness & 110 \\
\hline
\end{tabular}

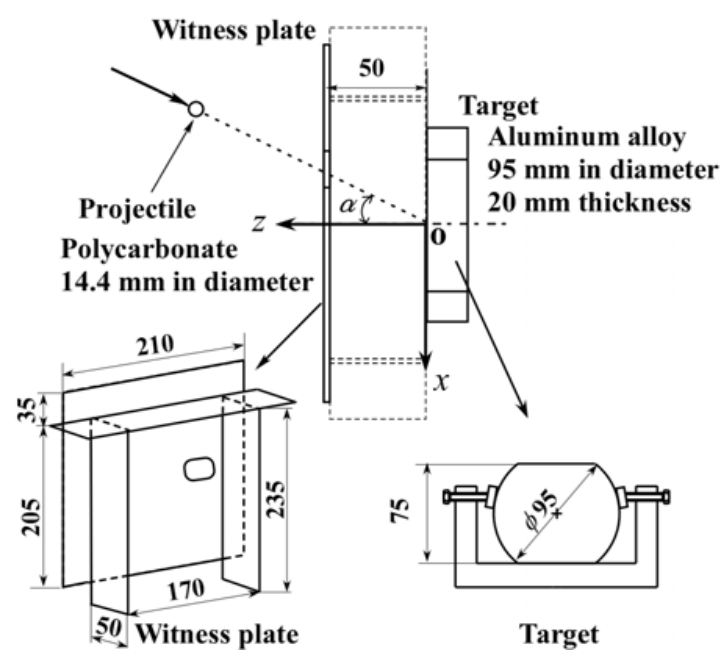

Fig. 1. Schematic of witness plates and target.

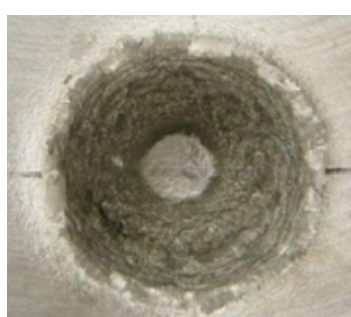

(a) $\alpha=0^{\circ}, 1.79 \mathrm{~km} / \mathrm{s}$

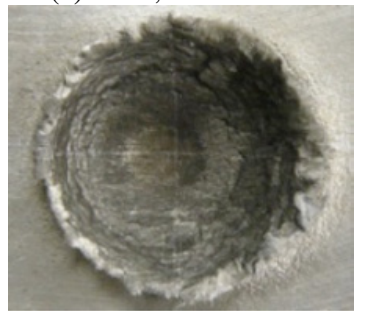

(c) $\alpha=22.5^{\circ}, 1.76 \mathrm{~km} / \mathrm{s}$

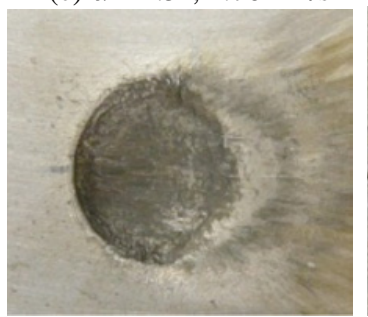

(e) $\alpha=45^{\circ}, 1.67 \mathrm{~km} / \mathrm{s}$

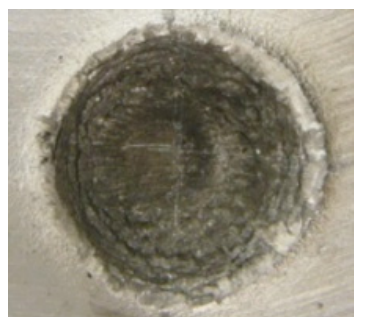

(b) $\alpha=15^{\circ}, 1.82 \mathrm{~km} / \mathrm{s}$

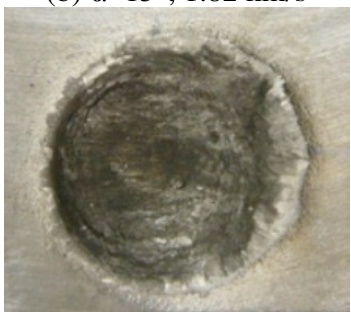

(d) $\alpha=30^{\circ}, 1.83 \mathrm{~km} / \mathrm{s}$

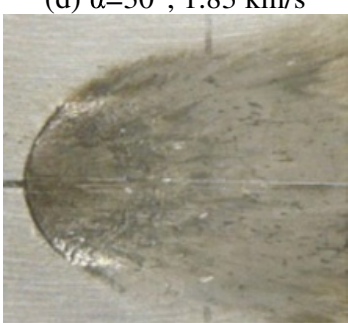

(f) $\alpha=60^{\circ}, 1.79 \mathrm{~km} / \mathrm{s}$

Fig. 2. Effect of impact angle on crater shape (impact velocity: approximately $1.8 \mathrm{~km} / \mathrm{s}$ ).

observed on the surface of the target. In 1993 Christiansen et al. [8] showed photographs of craters where a $1 \mathrm{~mm}$ aluminum sphere obliquely struck aluminum alloy 6061T6 at 6.5 to $7.0 \mathrm{~km} / \mathrm{s}$. Similar results to our own were

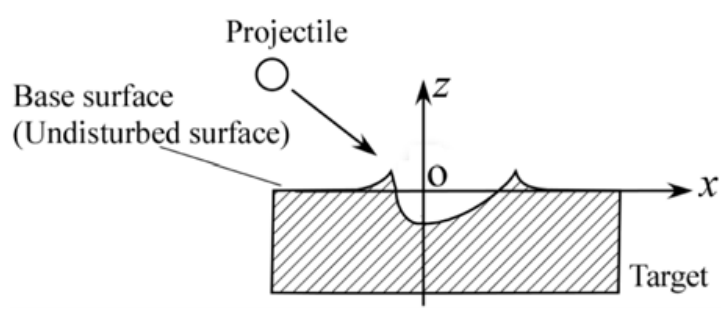

Fig. 3. Definition of crater shapes.

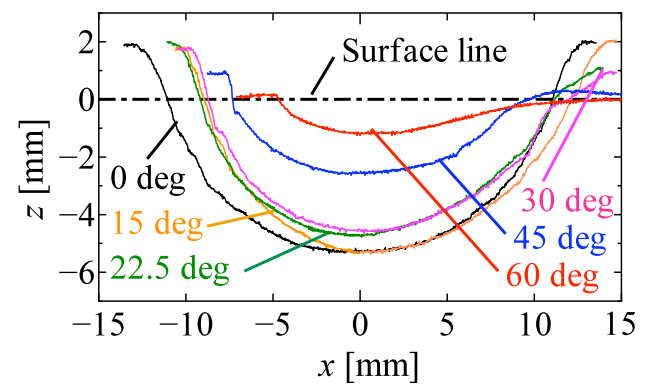

(a) Along the direction of the projectile flight path

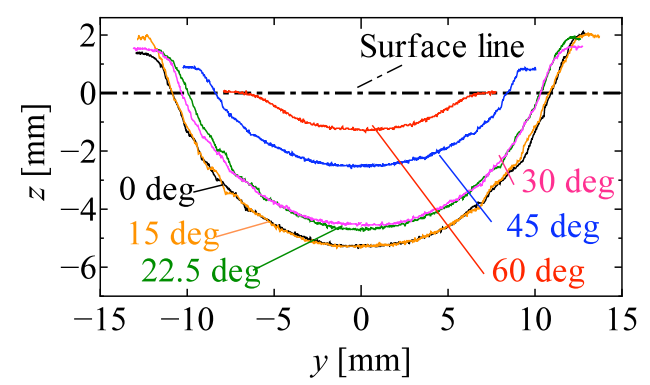

(b) Perpendicular to the direction of projectile flight path

Fig. 4. Schematic of witness plates and target.

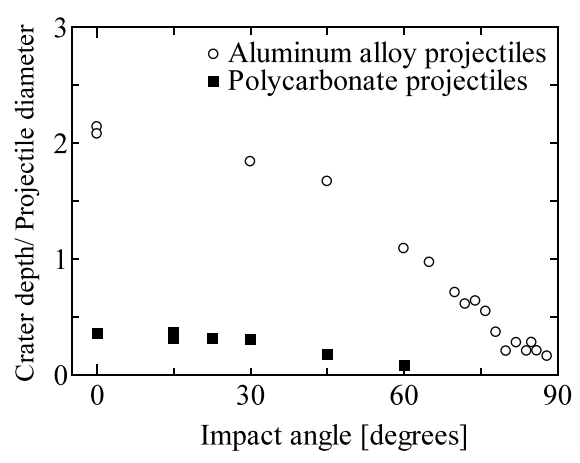

Fig. 5. Effect of impact angle on crater depth.

observed. However, in their paper, even at $70^{\circ}$, the crater lips were clearly observed. The crater was elliptical. At $76^{\circ}$, the main crater became more multi-put like. These results were different from ours.

Figures 4(a) and (b) show a comparison of the crater shapes (along the projectile flight path and perpendicular to the projectile flight path) at different impact angles. The crater shape was defined assuming an undisturbed surface, as shown in Fig. 3. The deepest point of each crater shape was oriented to the zero of the $x$ axis. As the impact angle increased, the entry side became steeper and the exit side became more moderate. Christiansen et al. reported that 


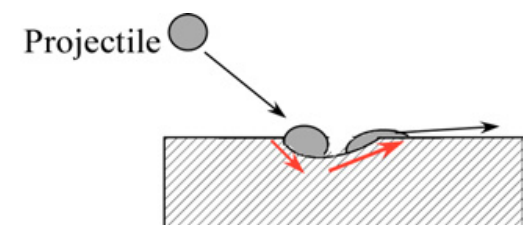

Fig. 6. Photographs of ejecta fragments collected from chamber.

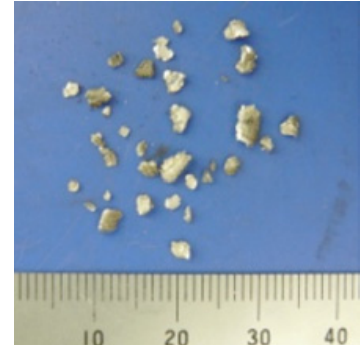

(a) $\alpha=0^{\circ}, 1.79 \mathrm{~km} / \mathrm{s}$

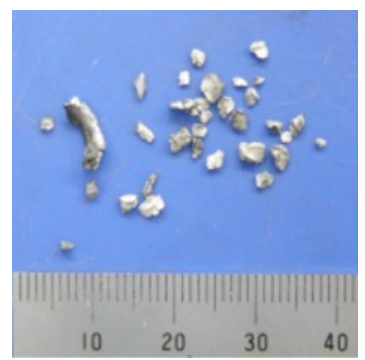

(c) $\alpha=22.5^{\circ}, 1.76 \mathrm{~km} / \mathrm{s}$

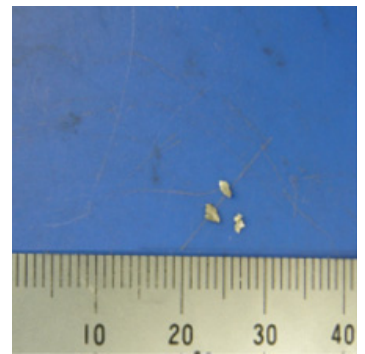

(e) $\alpha=45^{\circ}, 1.67 \mathrm{~km} / \mathrm{s}$

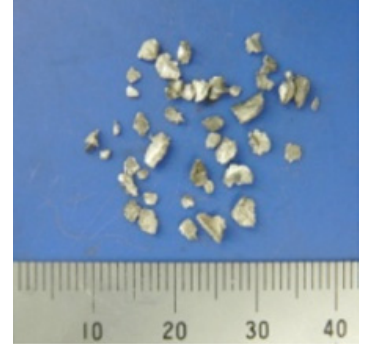

(b) $\alpha=15^{\circ}, 1.82 \mathrm{~km} / \mathrm{s}$

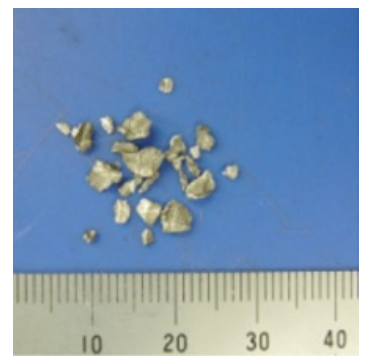

(d) $\alpha=30^{\circ}, 1.83 \mathrm{~km} / \mathrm{s}$

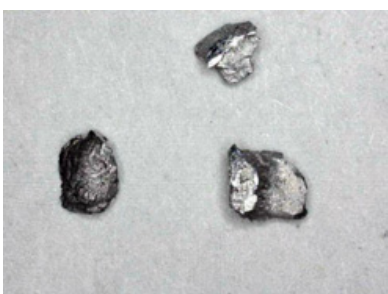

(f) Enlarged image of (a)
Fig. 7. Photographs of ejecta fragments collected from chamber.

the deepest part of the crater was on the entry side [8]. A similar tendency was observed in our experiments. It can be seen in Fig. 4(b) that when $\alpha=45^{\circ}$ and $60^{\circ}$ shallow crater shapes were created.

Figure 5 shows a comparison of the crater depth with the results of Christiansen et al. The results for polycarbonate projectiles decreased, showing a sharp decline over $30^{\circ}$, whereas the changed angle for aluminum alloy projectiles of Christiansen et al. was $45^{\circ}$. We predicted that the main reason for this variation was differences in the projectile material and impact velocity. It was predicted that polycarbonate projectiles would be easily deformed without deep penetration during an oblique impact, as shown in Fig. 6.

\subsection{Size and mass of ejecta fragments}

After the crater shapes were examined in Sect. 3.1 and the results were compared to those of an earlier study,

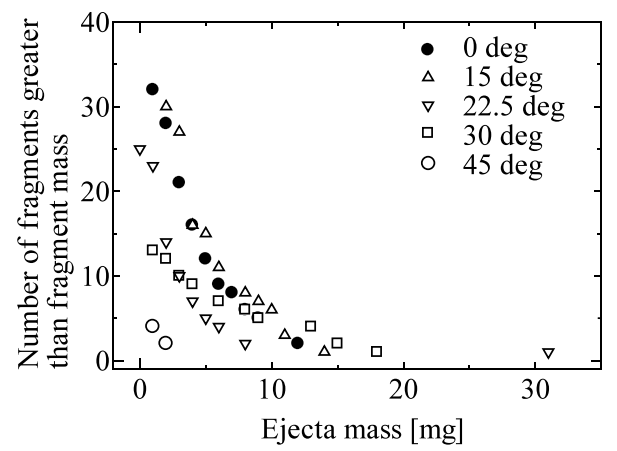

Fig. 8. Effect of impact angle on ejecta mass.

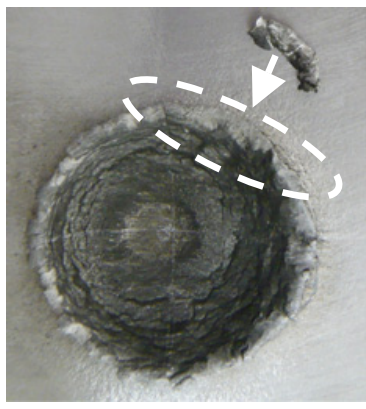

(a)

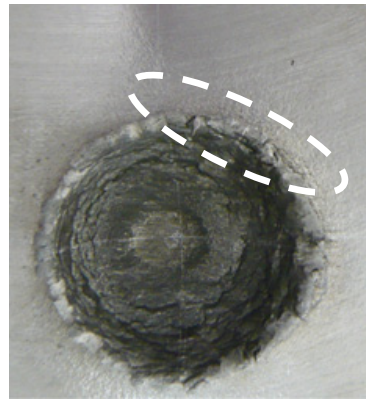

(b)
Fig. 9. Large ejecta fragments when $\alpha=22.5^{\circ}$.

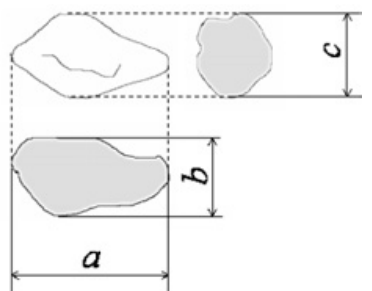

Fig. 10. Definition of ejecta fragment size.

the ejecta size and mass were examined as the main topic of this study. All the ejecta fragments from the targets, apart from the projectile fragments, were collected from the test chamber after the impact experiments. The collected ejecta are shown in Fig. 7. When $\alpha=60^{\circ}$, the visible ejecta fragments were not collected from the test chamber. Regardless of impact angle, the edges of the ejecta fragments were blunt.

Figure 8 shows the cumulative number distribution of ejecta mass, which means the number of ejecta fragments with a mass greater than the mass of ejecta fragments on the horizontal axis. The number distribution of ejecta mass did not show a clear trend. The mass at $45^{\circ}$ was not large and nor was the number of mass. A photograph of the largest ejecta fragment $(31 \mathrm{mg})$ at $22.5^{\circ}$ is shown on the upper right of Fig. 9(a) as well as Fig. 7(c). The large ejecta dovetails with a part of the ejecta lip, as shown in Fig. 9.

The size (length $a$, width $b$, thickness $c$ ) of the ejecta defined in Fig. 10, were measured. Figure 11 shows the cumulative number distribution of ejecta length, $a$. The ejecta length and the number of ejecta roughly decreased with increasing impact angle. The number of ejecta at $0^{\circ}$, $15^{\circ}$ and $22.5^{\circ}$ was almost the same. This tendency was 


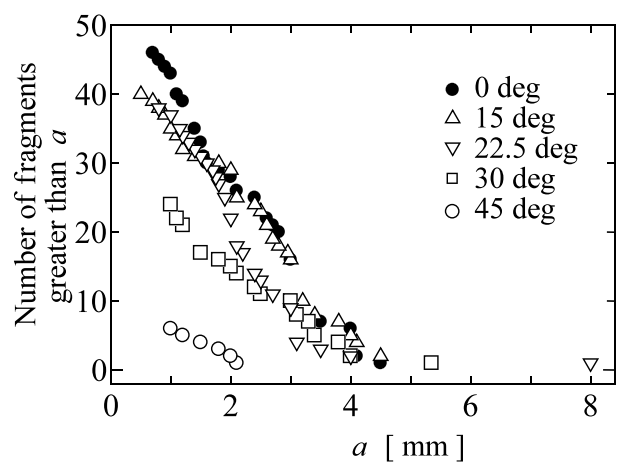

Fig. 11. Distribution of ejecta length.

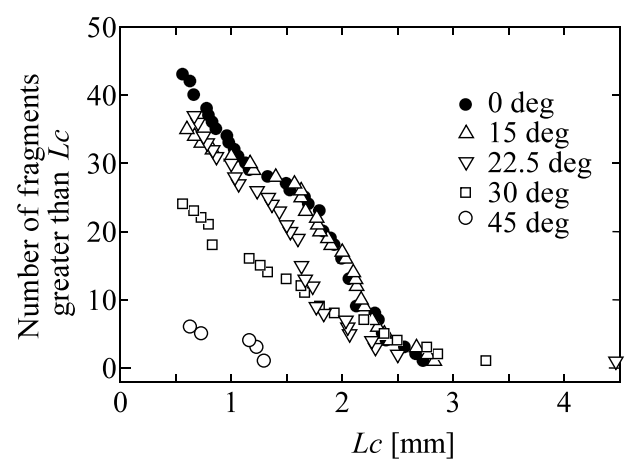

Fig. 12. Distribution of characteristic length.

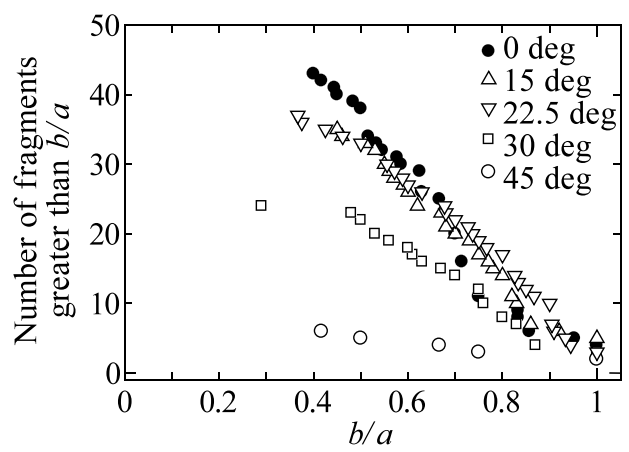

(a) Distribution of $b / a$

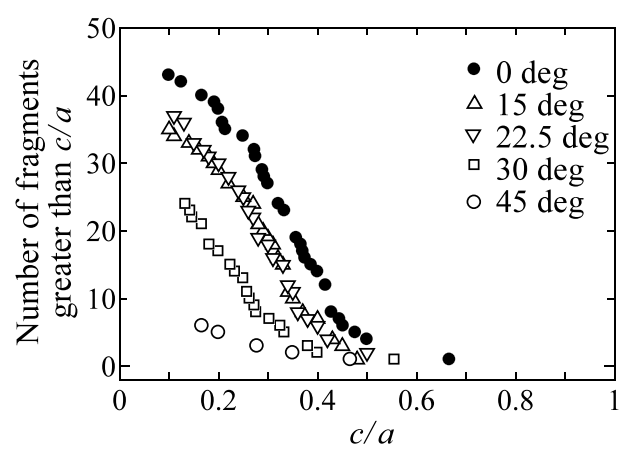

(b) Distribution of $c / a$

Fig. 13. Effect of impact angle on axial ratio of ejecta.

also observed in the results on the characteristic length of ejecta, $L c=(a+b+c) / 3$ [9], defined by NASA's breakup model, as shown in Fig. 12.

The axial ratios, $b / a$ and $c / a$, of ejecta fragments are shown in Fig. 13(a) and (b). A $b / a$ value close to 0

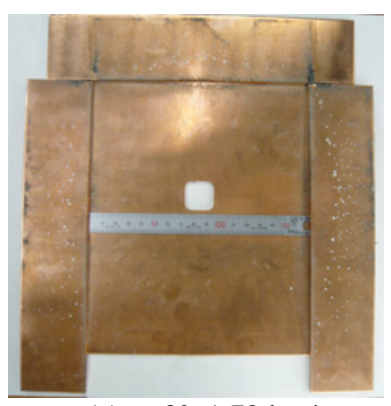

(a) $\alpha=0^{\circ}, 1.79 \mathrm{~km} / \mathrm{s}$

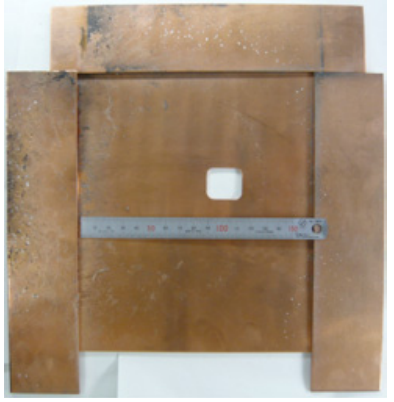

(c) $\alpha=22.5^{\circ}, 1.76 \mathrm{~km} / \mathrm{s}$

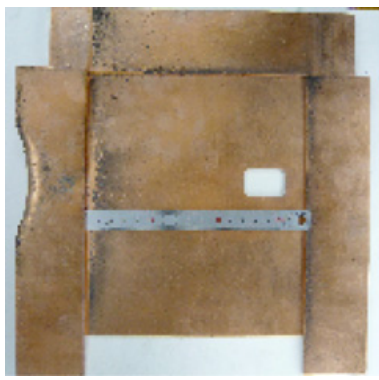

(e) $\alpha=45^{\circ}, 1.67 \mathrm{~km} / \mathrm{s}$

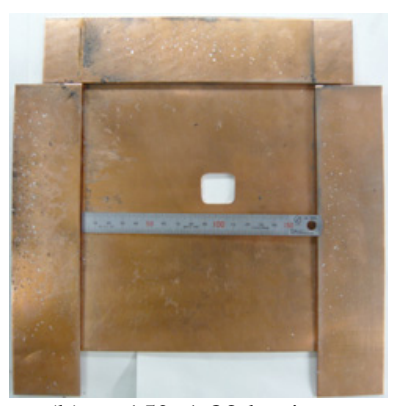

(b) $\alpha=15^{\circ}, 1.82 \mathrm{~km} / \mathrm{s}$

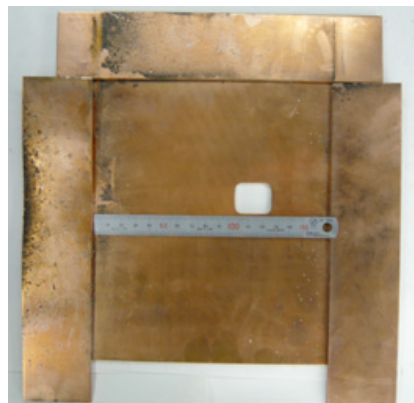

(d) $\alpha=30^{\circ}, 1.83 \mathrm{~km} / \mathrm{s}$

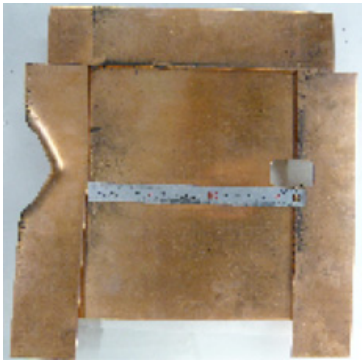

(f) $\alpha=60^{\circ}, 1.79 \mathrm{~km} / \mathrm{s}$
Fig. 14. Observation of indentations on witness plates.

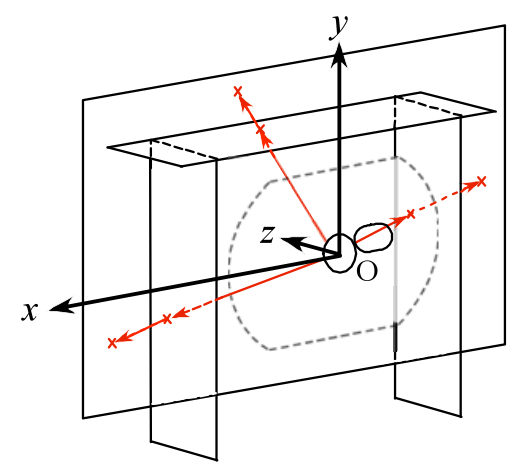

Fig. 15. Projection of indentations on witness plates.

indicates that the ejecta are slender. A $c / a$ value close to 1 indicates that they are thick, and a $c / a$ value close to 0 indicates that they are thin. It can be seen from Fig. 13(a) that when $\alpha=0^{\circ}, 15^{\circ}$ and $22.5^{\circ}$ the distributions were almost the same, and when $\alpha=30^{\circ}$ and $45^{\circ}$ there were not many slender ejecta fragments. Figure 13(b) shows that the thickness of ejecta fragments clearly decreased with increasing impact angle.

Compared with pure aluminum alloys, aluminum alloy 6061-T6 has larger yield stress, tensile strength and hardness, and smaller elongation at break. The authors group 


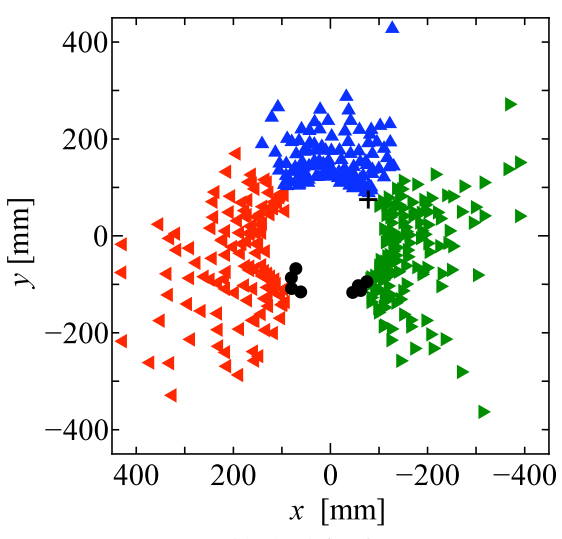

(a) $\alpha=0^{\circ}, 1.79 \mathrm{~km} / \mathrm{s}$

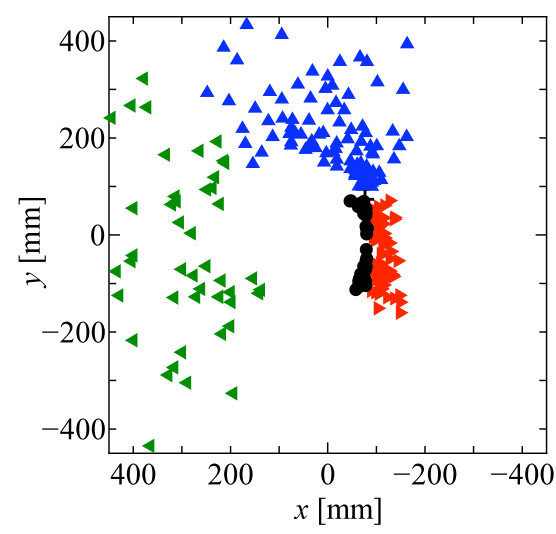

(c) $\alpha=22.5^{\circ}, 1.76 \mathrm{~km} / \mathrm{s}$

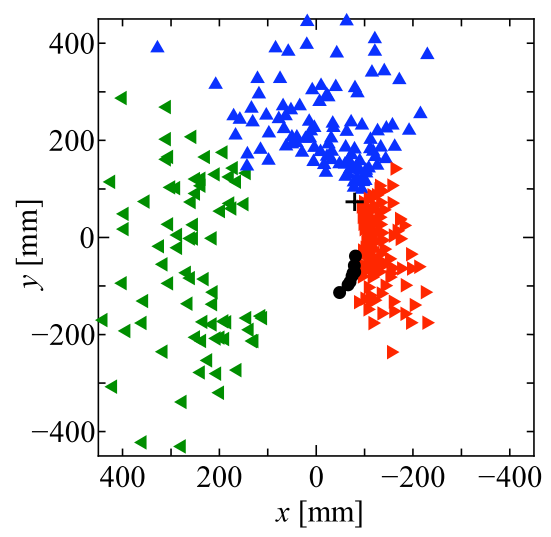

(b) $\alpha=15^{\circ}, 1.82 \mathrm{~km} / \mathrm{s}$

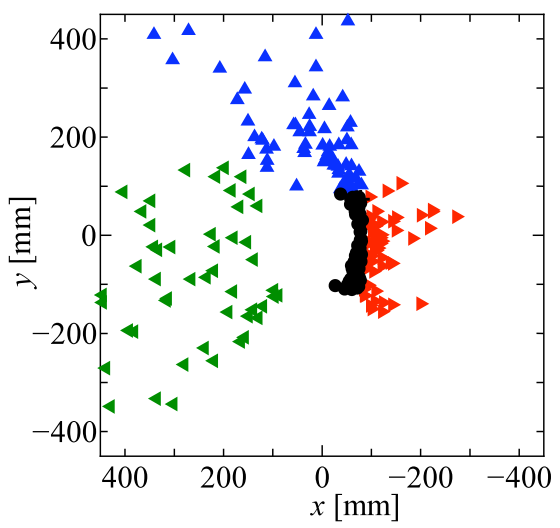

(d) $\alpha=30^{\circ}, 1.83 \mathrm{~km} / \mathrm{s}$

Fig. 16. Position of indentations on witness plates.

showed that the ejecta fragments of aluminum alloys 1100$\mathrm{O}$ and $-\mathrm{H}$ were thinner than those of 6061-T6 because they had such mechanical properties [7]. The ejecta fragments of even aluminum alloy 6061-T6 became thinner with increasing impact angles under our experimental conditions.

\subsection{Observation of indentations on witness plates}

Figure 14 shows the witness plates after the experiments. Because the indentations are small it is difficult to distinguish them in these images. However, the features of the indentations in the witness plates were almost the same at $0^{\circ}, 15^{\circ}, 22.5^{\circ}$ and $30^{\circ}$. Outside of the undamaged area, many indentations were observed, regardless of the impact angle. At $45^{\circ}$ and $60^{\circ}$, no clear indentations were observed.

As shown in Fig. 15, all indentations on the witness plates were projected on the largest witness plate $(50 \mathrm{~mm}$ away from targets; $z=50 \mathrm{~mm}$ ). Projected figures at $0^{\circ}$, $15^{\circ}, 22.5^{\circ}$ and $30^{\circ}$ are shown in Fig. 16(a)-(d). On the entry side (incident side), indentations were distributed into a narrow area, while on the reflection side indentations were distributed over a wide area.

Because the projected figures became distorted, in order to examine the scatter angle of ejecta fragments, the declination on a plane parallel to the target surface, $\theta$, and the angle from the axis perpendicular to the target surface, $\phi$, as shown in Fig. 17 were calculated using trigonometric

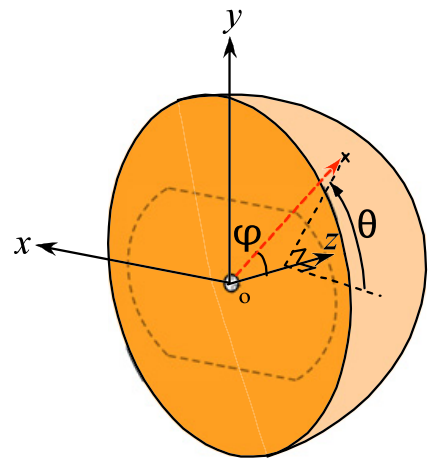

Fig. 17. Definition of scatter angles.

functions. The moving radius connecting the position of indentation to the closest edge of the crater was employed to calculate $\theta$ and $\phi$. Figure 18 shows the results at $0^{\circ}, 15^{\circ}$, $22.5^{\circ}$ and $30^{\circ}$. The projectiles came from the side of $\theta=0^{\circ}$ and were reflected toward $\theta=180^{\circ}$.

When the impact angle was $0^{\circ}$, the scatter angle was approximately $\phi=70-80^{\circ}$. When the impact angle was $15^{\circ}$, on the side of $\theta=0^{\circ}$ the scatter angle was near $\phi=70^{\circ}$, and on the other side $\phi$ was $80^{\circ}$. When the impact angles were $22.5^{\circ}$ and $30^{\circ}$ on the side of $\theta=0^{\circ}$, the scatter angle was $\phi=60^{\circ}$ and on the other side $\phi$ was $90^{\circ}$. The scatter angle depended on the impact angle. We predicted the scatter behavior of asymmetry shown in Fig. 19. 


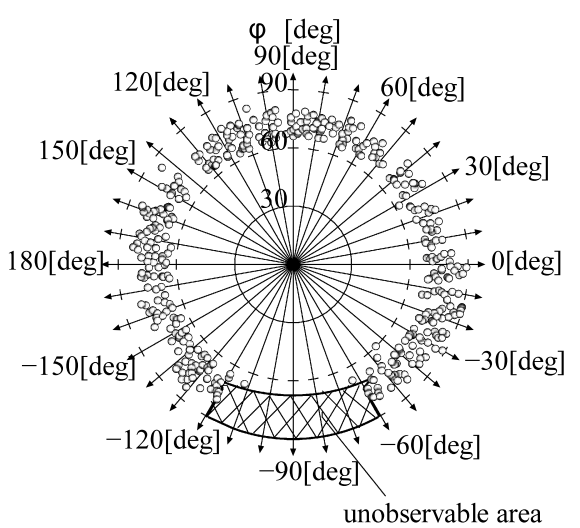

(a) $\alpha=0^{\circ}, 1.79 \mathrm{~km} / \mathrm{s}$

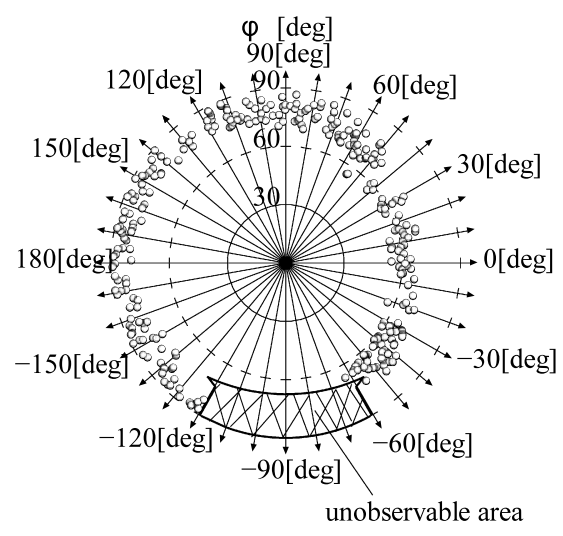

(c) $\alpha=22.5^{\circ}, 1.76 \mathrm{~km} / \mathrm{s}$

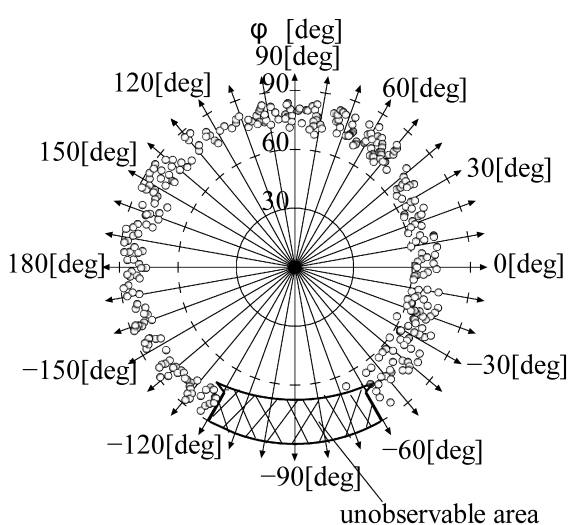

(b) $\alpha=15^{\circ}, 1.82 \mathrm{~km} / \mathrm{s}$

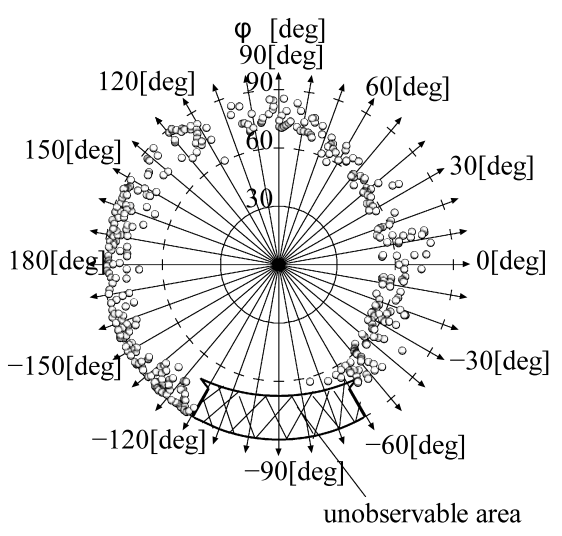

(d) $\alpha=30^{\circ}, 1.83 \mathrm{~km} / \mathrm{s}$

Fig. 18. Position of indentations on witness plates.

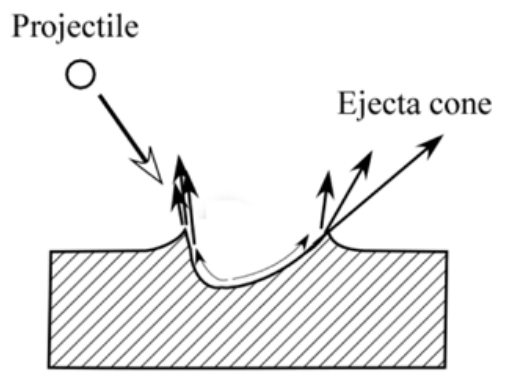

Fig. 19. Schematic representation of scatter of ejecta.

\section{Conclusions}

The impact angle of the projectiles clearly affected the crater shape, as expected. The trend was similar to the results of Christiansen et al. [8]. The impact angle also affected the ejecta mass, ejecta size and scatter angle. However, the effect at $15^{\circ}$ and $22.5^{\circ}$ was not large. When the impact angles were $30^{\circ}$ and $45^{\circ}$, the effect was clearly confirmed. The impact angle clearly affected the axial ratio of ejecta fragments, $c / a$.

\section{References}

1. O.L. Valerio-Flores, L.E. Murr, V.S. Hernandez, S.A. Quinodes, J. Mater. Sci. 39 (2004)

2. V.S. Hernandes, L.E. Murr, I.A. Anchondo, Int. J. Impact Eng. 32 (2006)

3. International Organization for Standardization, ISOCD-11227

4. K. Sugahara, K. Aso, Y. Akahoshi, T. Koura, Y. Narumi, Proc. 60th Int. Astronautical Cong., (2009)

5. J.M. Siguier, J.C. Mandeville, Proc. IMechE G 221 (2007)

6. D. Numata, T. Kikuchi, M. Sun, K. Kaiho, K. Takayama, Proc. 26th Int. Symp. Shock Waves, (2007)

7. M. Nishida, H. Kato, K. Kuzuya, K. Hayashi, S. Hasegawa, Proc. ICHSIP-29 (2010)

8. E.L. Christiansen, E. D. Cykowski, J. Ortega, Int. J. Impact Eng. 14 (1993)

9. N.L. Johnson, P.H. Krisko, J.-C. Liou, P.D. AnzMeador, Adv. Space Res., 28 (2001) 